\title{
Hunger Games: Associations between Core Eating Disorder Symptoms and Responses to Rejection by Peers during Competition
}

\author{
Sarah E. Schell, BSc ${ }^{1}$, Iulia Banica, BSc ${ }^{1}$, Anna Weinberg, $\mathrm{PhD}^{1}$, \& Sarah E. Racine, $\mathrm{PhD}^{1}$ \\ ${ }^{1}$ Department of Psychology, McGill University, Montreal, Canada
}

Correspondence regarding this manuscript may be sent to:

Sarah E. Racine

Department of Psychology

$2001 \mathrm{McGill}$ College, 7th floor

Montreal, QC

H3A $1 \mathrm{G} 1$

Telephone: 514-398-3649

Fax: 514-398-4896

Email: sarah.racine@mcgill.ca

Word count: $4923 / 4500$

Abstract: $248 / 250$

Tables: $2 / 2$ 
Data Availability Statement: The data that support the findings of this study are openly available in Open Science Framework at https://osf.io/jdvu2/?view_only=bef08068c156456799059cabdc8eccf0

Acknowledgements: This study was supported by a Canadian Institutes for Health Research (CIHR) grant (PJT-156079) awarded to Drs. Sarah Racine and Anna Weinberg, CIHR Tier 2 Canada Research Chair Awards awarded to Drs. Sarah Racine and Anna Weinberg, and Fonds de Recherche du Québec - Société et Culture doctoral fellowships awarded to Sarah Schell and Iulia Banica.

Conflict of Interest: The authors declare that there is no conflict of interest. 


\begin{abstract}
Objective: Individuals with eating disorder (ED) symptoms are sensitive to social threat and report maladaptive interpersonal styles that may contribute to and exacerbate negative evaluation from others. Research in this area has relied primarily on self-report. The current study examined associations between behavioral responses to social threat and core ED symptoms using a behavioral paradigm. Based on previous findings that individuals with binge eating report being more reactive and confrontational, whereas individuals with dietary restriction tend to be more submissive and avoidant of conflict, we hypothesized that binge eating would be associated with a greater tendency to retaliate against rejection perpetrators, whereas dietary restriction would be associated with a lower tendency to retaliate when rejected.
\end{abstract}

Method: Undergraduate women $(N=132)$ completed a self-report measure of ED symptoms and participated in an online "Survivor"-type game in which they voted to either accept or reject computerized coplayers, while also receiving acceptance or rejection feedback from others.

Results: Neither ED symptom was associated with how often participants retaliated against coplayers who rejected them. However, dietary restriction was related to more rejection votes overall (i.e., the tendency to reject others regardless of how others voted).

Discussion: Findings suggest that individuals with dietary restriction may rely on a maladaptive defensive strategy aimed at pre-empting rejection, or alternatively, have difficulty shifting from habitual self-isolating behavior that results from over-involvement with restricting symptoms. Interventions targeting hypersensitivity to social threat or interpersonal flexibility may help reduce interpersonal stress and mitigate its impact on restricting symptoms. Keywords: Eating Disorder Symptoms; Binge Eating; Dietary Restriction; Eating Disorders; Interpersonal Stress; Interpersonal Behavior 
Interpersonal stress, particularly negative social evaluation, has been shown to influence symptoms of eating disorders (EDs; see Monteleone et al., 2018 for a review). ED symptoms, in turn, can elicit negative evaluations from others, creating a cycle of increasing interpersonal stress and worsening symptoms (Nielsen \& Bará-Carril, 2003; Rieger et al., 2010; Schmidt \& Treasure, 2006). Additionally, individuals with EDs may exhibit maladaptive interpersonal biases and cognitions, particularly with regards to social threat (i.e., challenges to social inclusion or connectedness, such as rejection and ostracism; Rieger et al., 2010; Schmidt \& Treasure, 2006). Specifically, women with EDs show attentional biases towards angry and rejecting faces and report increased negative affect following interpersonal stressors, compared to controls (Cardi et al., 2013; Harrison et al., 2010; Monteleone et al., 2018). However, little research has examined how individuals with EDs respond to people who are sources of social threat (i.e., those who evaluate them negatively). Examining how individuals with ED symptoms react towards others when negatively evaluated might further our understanding of the bidirectional association between interpersonal difficulties and ED symptoms.

To our knowledge, few studies have examined how individuals with ED symptoms respond towards people who judge them negatively. Studies using the Means-Ends Problem Solving Procedure (MEPS; Platt \& Spivack, 1975), in which participants are asked to generate solutions to hypothetical interpersonal problems (e.g., rejection), have found that binge eating disorder (BED), anorexia nervosa (AN), and drive for thinness are associated with ineffective interpersonal problem solving (Ridout et al., 2015; Sternheim et al., 2020; Svaldi et al., 2011). In another study using hypothetical scenarios, women with AN and healthy controls were shown videos of hypothetical job supervisors providing critical feedback, ranging in tone from warm to cold (Ambwani et al., 2016). Participants then selected among possible behavioral responses to 
the feedback, also ranging from warm to cold. Participants with AN selected significantly more cold behavioral responses (e.g., "act unsociable or emotionally inhibited"), even when they perceived the supervisor's feedback to be warm. These findings suggest that, when faced with negative evaluation, women with ED symptoms may behave in ways that further exacerbate social difficulties.

Findings on interpersonal style, anger expression, and hostility in individuals with EDs can also inform hypotheses. Individuals' responses to social threat are likely influenced by general interpersonal dispositions. For example, individuals who tend to be angry, hostile, and antagonistic are more prone to retaliating and acting aggressively when provoked with interpersonal conflict, compared to individuals lower on these traits (for a review, see Bettencourt et al., 2006). Research on interpersonal styles in EDs has shown that ED symptoms are associated with maladaptive dispositions that may influence how individuals respond to social threat, and these dispositions appear to differ across specific EDs and core symptoms (Arcelus et al., 2013).

When examining diagnostic groups, individuals with bulimia nervosa $(\mathrm{BN})$ score higher on measures of interpersonal conflict, hostility, and outwardly expressed anger, compared to individuals with AN and healthy controls (Arcelus et al., 2013; Fassino et al., 2001; Tiller et al., 1995). It is unclear whether these characteristics are associated with a particular symptom of BN (i.e., binge eating, compensatory behavior) or the entire symptom profile, although research on individuals with BED suggests that these patients are more outwardly angry, domineering, and cold, but also more submissive, compared to overweight and healthy controls (Brugnera et al., 2018, 2019; Fassino et al., 2003). Conversely, individuals with AN tend to be conflict averse, are 
less hostile, and prioritize others' feelings, compared to individuals with $\mathrm{BN}$ and controls (Arcelus et al., 2013; Carter et al., 2012; Geller et al., 2000; Tiller et al., 1995).

Only a few studies have examined associations between continuously measured ED symptoms and interpersonal styles in non-clinical samples. There is some support for the aforementioned findings, specifically that binge eating (though not purging) is associated with outwardly expressed anger and that oral control (similar to dietary restriction) is associated with lower hostility (MacLaren \& Best, 2009; Peñas-Lledó et al., 2004). However, one study found that scores on a composite measure of bulimic symptoms were not associated with hostility, while another found that they were associated with anger suppression rather than expression (MacLaren \& Best, 2009; Milligan \& Waller, 2000).

Overall, available evidence suggests that individuals with binge eating may be more prone to conflict, expressing anger, and hostility. Based on this, we can hypothesize that these individuals may retaliate when faced with negative evaluation. Conversely, individuals with dietary restriction appear to avoid conflict and upsetting others, and may be less likely to retaliate. Notably, these patterns are clearer when examining diagnostic groups, whereas there is more variability in research examining continuously-measured symptoms. Further, self-report findings in individuals with AN contradict the study by Ambwani et al. (2016), who found that individuals with AN favoured conflict-supportive rather than conflict-avoidant behavior. Ambwani and colleagues suggest that this discrepancy may be due to differences in measurement - it is possible that in hypothetical scenarios (such as the Ambwani and colleagues scenarios and the MEPS), participants behave differently than they do in real life, and that the more commonly used self-reports (e.g., The Inventory of Interpersonal Problems, Horowitz et al., 2003; The State-Trait Anger Expression Inventory; Spielberger, 1996) better reflect typical 
behavior. However, self-report measures are vulnerable to desirable responding and inaccurate recall of past events. Examining actual interpersonal behavior in response to social threat using a non-hypothetical social interaction paradigm is warranted and may extend our understanding of the associations between interpersonal difficulties and ED symptoms.

The current study examined associations between responses to rejection, a particularly salient form of social threat shown to have negative emotional and behavioral consequences (Gerber \& Wheeler, 2009), and core ED symptoms (i.e., binge eating and dietary restriction). We used a novel interpersonal paradigm in which participants competed against coplayers (who were, unknown to participants, computer generated) in a realistic, computerized "Survivor"-type game (i.e., one in which participants vote to keep or oust one another from the game). Unlike self-report measures and hypothetical scenarios, this task allowed us to examine objective behavioral responses to subjectively "real" rejection. Our task also included a measure of participants' liking of coplayers, allowing us to examine effects independent of social anhedonia, which may affect behavioral responses to rejection and is found to be elevated in individuals with EDs (Harrison et al., 2014; Tchanturia et al., 2012).

Given findings suggesting that individuals with BN, BED, and binge eating may be more conflict-prone, hostile, and outwardly angry, whereas individuals with AN and dietary restriction may be more avoidant of conflict and upsetting others (e.g., Arcelus et al., 2013; Peñas-Lledó et al., 2004; Tiller et al., 1995), we hypothesized that binge eating would be associated with a greater tendency to retaliate against rejection perpetrators, whereas dietary restriction would be associated with a lower tendency to retaliate when rejected. Specifically, we hypothesized that binge eating would moderate the association between the feedback participants received from coplayers in the previous round (i.e., whether or not coplayers rejected them) and how 
participants voted for coplayers on the subsequent round, such that higher binge eating scores would be associated with a greater likelihood of participants voting to reject coplayers who rejected them in the previous round. Conversely, we hypothesized that dietary restriction would moderate the association between feedback received and subsequent voting, such that higher dietary restriction scores would be associated with a lower likelihood of participants voting to reject coplayers who rejected them in the previous round.

\section{Method}

\section{Participants}

Participants were 132 women who were either students enrolled at a North American university or members of the local community. Participants ranged in age from 18 to 29 years ( $M$ $=20.69, S D=2.24)$ and came from a range of ethnic/racial backgrounds: $49.6 \%$ identified as Caucasian, $15.7 \%$ as Chinese, $3.1 \%$ as South Asian, 3.1\% as Arab, 2.4\% as Japanese, 1.6\% as Korean, $1.6 \%$ as Latin American, $0.8 \%$ as Black, 0.8\% as Southeast Asian, $0.8 \%$ as West Asian, $14.2 \%$ as multiracial, $3.1 \%$ as other, and $2.4 \%$ preferred not to indicate ethnicity. Participants were recruited via a research participant pool organized by the authors' institution, posters around campus and the community, and online advertisements for a study on how brain activity relates to emotion and personality. Inclusion criteria were being over 18 years, female, and able to understand English. Students were offered either course credit or financial compensation for participation and community members were offered financial compensation. Five participants were excluded: two for incorrectly responding to an attention-check question, two due to prior familiarity with the social interaction task used, and one for not completing the questionnaires. Thus, the final sample size was 127 participants.

\section{Procedure}


Data for this manuscript were obtained from a larger study examining neural processing of social, monetary, and food reward. Prior to their laboratory visit, participants completed online questionnaires, including the EPSI among several other measures. At the start of the laboratory visit, participants were given information about the five reward tasks to be completed (n.b. the order of the first three tasks was random, whereas the order of the last two was the same for everyone), including the Island Getaway task, and informed consent was obtained.

Participants' profiles for the Island Getaway task were created (see below) and electroencephalogram (EEG) sensors were attached (n.b. EEG data were not analysed for the purpose of this study). Participants completed tasks in a sound-proof booth, with research assistants remaining outside the booth but in the experiment room.

Following task completion, participants rated the believability of the Island Getaway task and were debriefed about the computerized nature of the task.

Only study components relevant to the current research question are described in this manuscript. Data obtained from these components have not been included in other analyses or publications stemming from this study.

Island Getaway Task (modified from task described in Kujawa et al., 2014). The Island Getaway task is a social interaction paradigm in which participants are told they are playing a "Survivor" type game with 11 peers in other laboratories across Canada and the United States who are, in fact, computer-generated. The code for the original task can be found at http://arfer.net/projects/survivor. The modified version of the task differs from the original in several ways, including the modification of coplayer profiles to better represent the target age group, the inclusion of "ally" and "enemy" coplayers (see below), and the addition of liking ratings of coplayers. 
Before starting, participants are told that they will be playing a game consisting of six rounds in which players travel across Hawaiian islands. Each round, players vote to indicate whether they would like each coplayer to continue on the journey or be "kicked out" of the game. Participants are told that whoever receives the most "kick out" votes after each round will be eliminated, and that the goal of the game is to be one of six players to arrive at the final island. In reality, the task is programmed so that participants always make it to the final island.

Prior to beginning the task, participants have a headshot picture taken, answer demographic questions to create a player profile, and are shown their coplayers' profiles. Coplayers are six men and five women so that overall gender distribution in the game, including the participant, is equal. Coplayers' photographs and information are randomly combined each time the task is run (with the exception that images are matched to gender-typical first names), in order to reduce any systematic effects of coplayer on participant behavior.

Each round, when shown a coplayer's profile (presented in random order), participants vote to keep or kick out that coplayer. They are told that they are voting simultaneously with the coplayer displayed on their screen and will see the coplayer's vote once both finish voting. The coplayer profile is displayed until participants enter their vote. The message "Waiting for [coplayer name] to vote..." is displayed if participants vote faster than a simulated voting time (Kujawa et al., 2014). Rejection feedback (a red "thumbs down") or acceptance feedback (a green "thumbs up") from the coplayer is displayed. Participants are then asked to enter liking ratings for that coplayer. In the first round, participants are required to vote to kick out at least three coplayers and to keep at least three coplayers. In subsequent rounds, they may vote however they choose. 
After participants complete voting for a specific round, they are shown the coplayer who received the most "kick out" votes and are told this player was eliminated. With each round, participants expand their profiles by responding to a free-response question (e.g., "Who do you most admire?"). Coplayers' responses are visible on their profiles during subsequent voting rounds. In total, there are 51 voting/feedback trials across six rounds and task completion takes approximately 25 minutes.

To increase believability, four coplayers vote reciprocally with participants - that is, two coplayers vote to accept the participants on the first two rounds ("allies"), and two vote to reject on the first two rounds ("enemies"). Allies and enemies are selected systematically: they are the first and third coplayers that participants vote to keep and reject, respectively. All four coplayers then vote reciprocally on all subsequent rounds. The remaining coplayers' votes are programmed so that participants receive approximately half acceptance and half rejection feedback. In the current study, participants received an average of 24 "kick out" votes and 27 "keep" votes.

\section{Measures}

Coplayer Liking Ratings. After entering their vote for a given coplayer, participants are asked to use a visual analogue scale to rate how much they like that coplayer $(1=$ not at all, $9=$ extremely).

Believability Rating. Prior to debriefing, participants were verbally asked "On a scale from 1 to 5 , where 1 is "not at all" and 5 is "completely", how much did you believe you were playing against other lab participants in real time?'. Participants reported moderate belief that they were playing against real peers $(M=2.97 ; S D=1.30)$. We chose not to exclude participants based on belief ratings, given previous research demonstrating that social exclusion paradigms evoke 
negative reactions even when participants know that they are interacting with a computer or trained confederates (Zadro et al., 2004).

Eating Pathology Symptoms Inventory (EPSI; Forbush et al., 2013). The EPSI is a 45-item self-report questionnaire designed to measure ED symptoms via eight subscales. Using a fivepoint scale $(0=$ never to $4=$ very often $)$, participants rate the frequency of various symptoms over the preceding four weeks. The 8-item Binge Eating and 6-item Restricting subscales were used. In student samples, the Binge Eating and Restricting subscales have demonstrated adequate internal consistency $(\alpha \mathrm{s}=.83)$, good test-retest reliability over two-to-four weeks $(r \mathrm{~s}=.71-.75)$, and excellent convergent and discriminant validity (Forbush et al., 2013). Internal consistency estimates in the current study were good for Binge Eating $(\alpha=.82)$ and Restricting $(\alpha=.87)$ subscales.

Body Mass Index (BMI). BMI was calculated based on participants's self-reported height and weight using the standard formula ((weight in pounds)/(height in inches $\left.\left.{ }^{2}\right)\right)^{*} 703$. Participant BMIs ranged from underweight to obese $(M[S D]=21.40[3.08]$; range $=15.33-33.25)$.

\section{Statistical Analyses}

We used generalized linear mixed models (GLMM) to examine whether ED symptoms moderated associations between the feedback participants received during the previous round of the Island Getaway task and their vote to accept or reject coplayers during the following round. Voting response (level 1) was nested within the 127 participants as well as the six rounds of the Island Getaway task. Participant and round were crossed at level 2, since every participant voted in every round. Participants' voting behavior was entered as the dichotomous outcome variable $(0=$ accept, $1=$ reject $)$. Feedback received during the previous round (level 1$)$, ED symptom (i.e., binge eating or restricting scores; level 2), and the interaction between feedback and 
symptom were entered as fixed effects. Intercepts and slopes for participant and round were entered as random effects. We included liking of coplayers during the previous round as a covariate in order to examine effects independent of participants' general tendency to like others (results are described with and without liking as a covariate). Only data from rounds 2 to 6 were used as there was no previous round feedback associated with round 1 voting behavior. Separate models were constructed for binge eating and dietary restriction in SPSS (24.0; SPSS Inc.).

\section{Results}

\section{Descriptive Statistics}

Based on guidelines proposed by Kim (2013) regarding cutoffs for standardized skewness scores, and results of Kolmogorov-Smirnov (KS) tests, a square-root transformation was used to correct for the positive skew of binge eating $\left(Z_{\text {skewness }}=4.03 ; K S=0.10, p=.004\right)$ and restricting $\left(Z_{\text {skewness }}=3.69 ; K S=0.12, p<.001\right)$ scores. All continuous variables were standardized prior to analyses.

Descriptive statistics and zero-order correlations are presented in Table 1. On average, participants voted to reject coplayers $39 \%$ of the time. Binge eating and restricting scores were comparable to established norms for undergraduate women (Binge Eating: $M_{\text {norm }}=8.92, S D=$ $4.52 ; t(278)=0.34, p=.74$; Restricting: $M_{\text {norm }}=5.55, S D=4.47 ; t(278)=1.44, p=.15$; Forbush et al., 2013).

\section{GLMM Results}

Table 2 presents GLMM results. Participants were more likely to vote to reject coplayers with each successive round, more likely to vote to reject a coplayer who had rejected them in the previous round, and less likely to vote to reject coplayers whom they liked in the previous round. Binge eating scores were not significantly associated with the likelihood of voting to reject 
coplayers, and the interaction between voting feedback received during the previous round and binge eating was non-significant. The pattern of results did not differ when previous round liking was removed as a covariate.

In contrast, there was a main effect of restricting scores on voting behavior, such that higher restricting scores were associated with a greater likelihood of voting to reject coplayers. The interaction between feedback received during the previous round and restricting was nonsignificant. When previous round liking was removed as a covariate, there was no longer a statistically significant main effect of dietary restriction, although the change in effect size was small (partial $R^{2}$ decreased from 0.09 to 0.07 ).

\section{Post-hoc analyses}

To rule out alternative explanations (i.e., explore whether other factors influenced the association between ED symptoms and voting behavior), post-hoc analyses examined: 1) the effect of ED symptoms on the association between coplayer voting and participants' liking of coplayers (i.e., do ED symptoms influence the extent to which previous voting is associated with coplayer liking?); 2) whether the effect of ED symptoms on voting behavior or liking ratings differed as a function of coplayer gender (i.e., are effects more pronounced for coplayers of a given gender?); 3) whether results reflect social learning difficulties (i.e., did participants higher in ED symptoms not understand how to appropriately play the game or differentiate between allies and enemies?); 4) whether BMI influenced our findings (i.e., do results differ based on participants' weight status?); and 5) whether the extent to which participants believed that they were playing against real coplayers influenced results (i.e., do results differ for participants who did not believe the task?). 
The only analysis that changed our pattern of results was the inclusion of BMI as a covariate (see Supplemental Material). Including BMI as a covariate strengthened the effect of binge eating (change in Partial $R^{2}$ from 0.02 to 0.05 ), and attenuated the effect of dietary restriction (change in Partial $R^{2}$ from 0.09 to 0.05 ), on the tendency to reject co-players.

\section{Discussion}

The current study examined associations between core ED symptoms and interpersonal behavior following rejection by "peers" in a computerized, "Survivor"-type game. We hypothesized that binge eating would be associated with a greater tendency to retaliate in response to rejection, whereas dietary restriction would be associated with a lower retaliation tendency. We found that neither binge eating nor dietary restriction moderated the association between previous coplayer votes (i.e., how coplayers voted for participants in the preceding round) and participant voting (i.e., how participants voted for coplayers in the current round), indicating that the tendency to retaliate against coplayers did not differ based on these ED symptoms. Interestingly, however, restricting scores were associated with a greater tendency to reject coplayers overall, regardless of how coplayers previously voted. Thus, individuals with dietary restriction may engage in maladaptive interpersonal behavior that is independent of how they are treated by others.

Contrary to hypotheses and previous findings suggesting that reactions to social threat may differ across ED symptoms (e.g., Arcelus et al., 2013; Fassino et al., 2001; Tiller et al., 1995), the extent to which rejection was associated with retaliation did not vary based on ED symptom severity. The discrepancy between our findings and those derived from self-reports of interpersonal styles may be explained by inaccurate self-reporting. Individuals with dietary restriction may describe their interpersonal styles as more socially desirable than they actually 
are, and individuals with binge eating may describe their interpersonal styles as more problematic. However, it is unclear why participants with dietary restriction would present themselves more positively compared to participants with binge eating, given that both groups exhibit high levels of self-presentation and socially-prescribed perfectionism (which could influence positive self-descriptions; Bardone-Cone, 2007; Hewitt et al., 1995; Stoeber et al, 2017) as well as high levels of self-criticism (which could influence negative self-descriptions; Duarte et al., 2017; Fennig et al., 2008). Alternatively, the online nature of the Island Getaway task may have influenced participants' behavior, such that they behaved differently than they would face-to-face. Indeed, individuals are less inhibited and display more antisocial behavior online compared to in-person (e.g., Lapidot-Lefler \& Barak, 2012; Suler, 2004). Given the recent rise in social media use, research investigating whether maladaptive interpersonal styles traditionally associated with EDs are consistent across modalities (i.e., in-person vs. online) is warranted. Finally, the majority of the extant research used to formulate our hypotheses was conducted using clinical samples with distinct EDs, whereas we measured ED symptoms continuously in a non-clinical sample. It may be that interpersonal behavior differs at higher levels of ED symptoms and/or that individual ED symptoms relate differently to interpersonal behavior than ED diagnoses. As such, our ability to extend our findings beyond individual symptoms to broader diagnostic groups is limited.

Interestingly, while we found that neither ED symptom was associated with the likelihood of retaliation, dietary restriction was associated with a greater tendency to reject coplayers, regardless of how coplayers voted. In other words, the behavior of participants with dietary restriction appeared to be independent of, and not always in line with, feedback received from coplayers. This finding is similar to that of Ambwani et al. (2016) where women with AN 
were more likely to select cold responses to interpersonal feedback, even when the feedback was perceived as warm. It is also in line with, and offers a possible explanation for, findings from studies examining social networks in relation to ED symptoms, which have shown that dietary restriction and restraint, but not binge eating, are associated with fewer friendships in young adults and adolescents (Forney et al., 2019; Rayner et al., 2013). Of note, covarying BMI attenuated the effect of dietary restriction, and strengthened the effect of binge eating, on the tendency to reject coplayers. This suggests that BMI may partially account for the effect of ED symptoms on the tendency to reject others, which is surprising given findings that, on its own, BMI is not associated with interpersonal problems (Blomquist et al., 2012; Carr \& Friedman, 2006).

Although our results do not allow us to draw conclusions about mechanisms linking dietary restriction and the tendency to reject others, we can speculate based on previous research. This finding might be explained by the association between dietary restriction and rejection sensitivity (i.e., being hyper-attuned and emotionally reactive to rejection; Cardi et al., 2013, 2017; Monteleone et al., 2018). Rejection-sensitive individuals are thought to demonstrate a maladaptive defensive strategy in which they attempt to prevent rejection by shunning others (Romero-Canyas \& Downey, 2005). Given the nature of the Island Getaway task (i.e., the explicit possibility of being rejected by coplayers), individuals with higher levels of dietary restriction may have become hyper-attuned to the threat of rejection, leading them to reject others before others could reject them. The tendency to push others away to avoid rejection may contribute to the significant interpersonal difficulties thought to influence the onset and maintenance of EDs (Cardi et al., 2018; Monteleone et al., 2018; Rieger et al., 2010; Wilfley et al., 2003) Although binge eating has also been associated with rejection sensitivity (Monteleone 
et al., 2018), there is some evidence that rejection sensitivity and interpersonal problems are more pronounced among individuals with AN (Cardi et al., 2013; Raykos et al., 2014).

An alternate explanation may be that individuals with dietary restriction exhibit maladaptive interpersonal habits related to their symptoms. In AN, as symptoms become more established, individuals become increasingly socially withdrawn, as their focus centers more on their ED and less on valued goals in other domains (Mulkerrin et al., 2016; Wildes \& Marcus, 2011). Further, individuals with AN tend to exhibit rigid behavior and inflexiblity across situations (Steinglass \& Walsh, 2006; Tchanturia et al., 2004). It may be that individuals with dietary restriction are used to behaving in ways that isolate them from others, and that in the Island Getaway Task, participants had difficulty shifting from this behavioral pattern. It is unclear, however, why this pattern would not also be observed for binge eating, given that both $\mathrm{BN}$ and BED have been associated with social withdrawal and lack of flexibility (Lobera et al., 2009; Roberts et al., 2007). One possibility, although entirely speculative, is that the discrepancy lies in social cognition. Whereas individuals with AN have difficulties considering the internal experiences of others (Russell et al., 2009), individuals with BN and BED, who show less pronounced deficits in social cognition (Aloi et al., 2017; DeJong et al., 2013), may be able to override their tendency to withdraw by considering the impact of their behavior on others.

The use of a computerized, "Survivor"-type game was a notable strength of our study. It allowed us to examine interpersonal responses to rejection in association with ED symptoms using a realistic behavioral paradigm, increasing the likelihood that results reflected participants' typical behavior. Additionally, the use of the Island Getaway task allowed us to examine retaliatory behavior as it occurred, rather than relying on retrospective reports that may be inaccurate. Given that coplayer profiles were randomized (i.e., participants did not all play 
against the same set of coplayers), we can also be confident that participants' behavior was associated with coplayer feedback (i.e., voting) rather than individual coplayer characteristics. There are also limitations to our methodology. First, the computerized task may not have adequately represented real-life stressors associated with ED symptoms. Participants competed against unknown "peers", and research in non-ED samples suggests that individuals react differently to rejection when perpetrated by strangers versus people they know, although there is conflicting evidence as to which has a more negative impact (Leary et al., 1998; Snapp \& Leary, 2001; Sommer et al., 2009). Further, while forming and maintaining alliances can be viewed as beneficial to succeeding in the Island Getaway task, alliances formed during competition might not be an ideal proxy for meaningful interpersonal relationships. Future research should examine associations between ED symptoms and retaliation towards close individuals (e.g., friends, family, romantic partners). Second, the task was computerized rather than in-person. While emotional responses to rejection appear to be consistent across modalities (Filipkowski \& Smyth, 2012), behavioral responses to rejection may differ with increased anonymity. Future research should aim to replicate the current findings using a less anonymous version of the task, for example, by having participants play the game on computers, but in the same room as coplayers. Third, we examined a non-clinical, student sample. It is possible that maladaptive interpersonal styles are not present in individuals with less severe symptoms, as evidenced by inconsistent findings on the association between interpersonal styles and ED symptoms in non-clinical samples (MacLaren \& Best, 2009; Milligan \& Waller, 2000). Replicating the current study in a clinical sample might help clarify the inconsistency between the current findings and extant literature. Finally, we did not ask participants to report their reasons for rejecting coplayers. 
Elucidating the motivation behind rejection would enhance our understanding of how characteristics of individuals with EDs promote interpersonal stress.

This study was, to our knowledge, the first to examine the association between core ED symptoms and interpersonal responses to rejection using a realistic behavioral paradigm. While our hypotheses were not supported, the finding that dietary restriction was associated with a greater tendency to reject coplayers overall provides insight into how interpersonal problems may develop and persist in individuals with dietary restriction. Rejecting others has the effect of limiting the possibility for development and growth of interpersonal relationships. Our findings suggest two possible avenues for intervention. First, modifying sensitivity to social threat may be helpful in reducing interpersonal stress in individuals with dietary restriction. A recent study demonstrated that positive interpretation bias training (i.e., increasing positive/realistic interpretations of ambiguous scenarios) lead to a reduction in negative interpretations of social scenarios and increased self-esteem in adolescents with AN (Cardi et al., 2019). Second, targeting rigidity might be helpful in promoting adaptive interpersonal behavior, rather than inflexible withdrawal patterns. A randomized control trial of cognitive remediation therapy in individuals with AN demonstrated that it was effective in increasing cognitive flexibility (Brockmeyer et al., 2014), but whether this treatment can impact social behavior is unclear. 


\section{References}

Aloi, M., Rania, M., Caroleo, M., De Fazio, P., \& Segura-García, C. (2017). Social cognition and emotional functioning in patients with binge eating disorder. European Eating Disorders Review, 25(3), 172-178. https://doi.org/10.1002/erv.2504

Ambwani, S., Berenson, K. R., Simms, L., Li, A., Corfield, F., \& Treasure, J. (2016). Seeing things differently: An experimental investigation of social cognition and interpersonal behavior in anorexia nervosa. International Journal of Eating Disorders, 49(5), 499-506. https://doi.org/10.1002/eat.22498

Arcelus, J., Haslam, M., Farrow, C., \& Meyer, C. (2013). The role of interpersonal functioning in the maintenance of eating. Clinical Psychology Review, 33(1), 1-17. https://doi.org/10.1016/j.cpr.2012.10.009

Bardone-Cone, A. M. (2007). Self-oriented and socially prescribed perfectionism dimensions and their associations with disordered eating. Behaviour Research and Therapy, 45(8), 1977-1986. https://doi.org/10.1016/j.brat.2006.10.004

Bettencourt, B. A., Talley, A., Benjamin, A. J., \& Valentine, J. (2006). Personality and aggressive behavior under provoking and neutral conditions: A meta-analytic review. Psychological Bulletin, 132(5), 751-777. https://doi.org/10.1037/0033-2909.132.5.751

Blomquist, K. K., Ansell, E. B., White, M. A., Masheb, R. M., \& Grilo, C. M. (2012).

Interpersonal problems and developmental trajectories of binge eating disorder. Comprehensive Psychiatry, 53(8), 1088-1095. https://doi.org/10.1016/j.comppsych.2012.05.003

Brockmeyer, T., Ingenerf, K., Walther, S., Wild, B., Hartmann, M., Herzog, W., Bents, H., \& Friederich, H. C. (2014). Training cognitive flexibility in patients with anorexia nervosa: A 
pilot randomized controlled trial of cognitive remediation therapy. International Journal of Eating Disorders, 47(1), 24-31. https://doi.org/10.1002/eat.22206

Brugnera, A., Carlucci, S., Compare, A., \& Tasca, G. A. (2019). Persistence of friendly and submissive interpersonal styles among those with binge-eating disorder: Comparisons with matched controls and outcomes after group therapy. Clinical Psychology and Psychotherapy, 26(5), 603-615. https://doi.org/10.1002/cpp.2385

Brugnera, A., Lo Coco, G., Salerno, L., Sutton, R., Gullo, S., Compare, A., \& Tasca, G. A. (2018). Patients with Binge Eating Disorder and Obesity have qualitatively different interpersonal characteristics: Results from an Interpersonal Circumplex study. Comprehensive Psychiatry, 85, 36-41. https://doi.org/10.1016/j.comppsych.2018.06.008

Cardi, V., Mallorqui-Bague, N., Albano, G., Monteleone, A. M., Fernandez-Aranda, F., \& Treasure, J. (2018). Social difficulties as risk and maintaining factors in anorexia nervosa: A mixed-method investigation. Frontiers in Psychiatry, 9. https://doi.org/10.3389/fpsyt.2018.00012

Cardi, V., Matteo, R. Di, Corfield, F., \& Treasure, J. (2013). Social reward and rejection sensitivity in eating disorders: An investigation of attentional bias and early experiences. World Journal of Biological Psychiatry, 14(8), 622-633. https://doi.org/10.3109/15622975.2012.665479

Cardi, V., Turton, R., Brazil, C., Harrison, A., Rowlands, K., Treasure, J., \& Hirsch, C. (2019). Training Rejection Interpretation in Eating disordeRs (TRIER): Preliminary findings of a feasibility study in adolescents with anorexia nervosa. Cognitive Therapy and Research, 43(6), 1109-1117. https://doi.org/10.1007/s10608-019-10034-х

Cardi, V., Turton, R., Schifano, S., Leppanen, J., Hirsch, C. R., \& Treasure, J. (2017). Biased 
Interpretation of Ambiguous Social Scenarios in Anorexia Nervosa. European Eating Disorders Review, 25(1), 60-64. https://doi.org/10.1002/erv.2493

Carr, D., \& Friedman, M. A. (2006). Body weight and the quality of interpersonal relationships. Social Psychology Quarterly, 69(2), 127-149. https://doi.org/10.1177/019027250606900202

Carter, J. C., Kelly, A. C., \& Norwood, S. J. (2012). Interpersonal problems in anorexia nervosa: Social inhibition as defining and detrimental. Personality and Individual Differences, 53(3), 169-174. https://doi.org/10.1016/j.paid.2012.02.020

DeJong, H., Van den Eynde, F., Broadbent, H., Kenyon, M. D., Lavender, A., Startup, H., \& Schmidt, U. (2013). Social cognition in bulimia nervosa: A systematic review. European Psychiatry, 28(1), 1-6. https://doi.org/10.1016/j.eurpsy.2011.07.002

Duarte, C., Matos, M., Stubbs, R. J., Gale, C., Morris, L., Gouveia, J. P., \& Gilbert, P. (2017). The impact of shame, self-criticism and social rank on eating behaviours in overweight and obese women participating in a weight management programme. PLoS ONE, 12(1), 1-14. https://doi.org/10.1371/journal.pone.0167571

Ethridge, P., Kujawa, A., Dirks, M. A., Arfer, K. B., Kessel, E. M., Klein, D. N., \& Weinberg, A. (2017). Neural responses to social and monetary reward in early adolescence and emerging adulthood. Psychophysiology, 54(12), 1786-1799. https://doi.org/10.1111/psyp.12957

Fassino, S., Abbate-Daga, G., Pierò, A., Leombruni, P., \& Rovera, G. G. (2003). Dropout from brief psychotherapy within a combination treatment in bulimia nervosa: Role of personality and anger. Psychotherapy and Psychosomatics, 72(4), 203-210. https://doi.org/10.1159/000070784

Fassino, S., Daga, G. A., Pierò, A., Leombruni, P., \& Rovera, G. G. (2001). Anger and 
personality in eating disorders. Journal of Psychosomatic Research, 51(6), 757-764.

https://doi.org/10.1016/S0022-3999(01)00280-X

Fennig, S., Hadas, A., Itzhaky, L., Roe, D., Apter, A., \& Shahar, G. (2008). Self-criticism is a key predictor of eating disorder dimensions among inpatient adolescent females. International Journal of Eating Disorders, 41(8), 762-765. https://doi.org/10.1002/eat.20573

Filipkowski, K. B., \& Smyth, J. M. (2012). Plugged in but not connected: Individuals' views of and responses to online and in-person ostracism. Computers in Human Behavior, 28(4), 1241-1253. https://doi.org/10.1016/j.chb.2012.02.007

Forbush, K. T., Wildes, J. E., Pollack, L. O., Dunbar, D., Luo, J., Patterson, K., Petruzzi, L., Pollpeter, M., Miller, H., Stone, A., \& Bright, A. (2013). Development and validation of the Eating Pathology Symptoms Inventory (EPSI). Psychological Assessment, 25(3), 859-878. https://doi.org/http://dx.doi.org/10.1037/a0032639

Forney, K. J., Schwendler, T., \& Ward, R. M. (2019). Examining similarities in eating pathology, negative affect, and perfectionism among peers: A social network analysis. Appetite, 137, 236-243. https://doi.org/10.1016/j.appet.2019.03.013

Geller, J., Cockell, S. J., Hewitt, P. L., Goldner, E. M., \& Flett, G. L. (2000). Inhibited expression of negative emotions and interpersonal orientation in anorexia nervosa. International Journal of Eating Disorders, 28(1), 8-19. https://doi.org/10.1002/1098108X(200007)28:1<8::AID-EAT2>3.0.CO;2-U

Gerber, J., \& Wheeler, L. (2009). On being rejected: A meta-analysis of experimental research on rejection. 4(5), 468-488. https://doi.org/10.1111/j.1745-6924.2009.01158.x

Harrison, A., Sullivan, S., Tchanturia, K., \& Treasure, J. (2010). Emotional functioning in eating 
disorders: Attentional bias, emotion recognition and emotion regulation. Psychological Medicine, 40(11), 1887-1897. https://doi.org/10.1017/S0033291710000036

Harrison, A, Mountford, V. A., \& Tchanturia, K. (2014). Social anhedonia and work and social functioning in the acute and recovered phases of eating disorders. Psychiatry Research, 218(1-2), 187-194. https://doi.org/10.1016/j.psychres.2014.04.007

Hewitt, P. L., Flett, G. L., \& Ediger, E. (1995). Perfectionism traits and perfectionistic selfpresentation in eating disorder attitudes, characteristics, and symptoms. International Journal of Eating Disorders, 18(4), 317-326. https://doi.org/10.1002/1098108X(199512)18:4<317::AID-EAT2260180404>3.0.CO;2-2

Horowitz, L. M., Alden, L. E., Wiggins, J. S., \& Pincus, A. L. (2003). Manual for the Inventory of Interpersonal Problems. Mind Garden Inc.

Kim, H.-Y. (2013). Statistical notes for clinical researchers: assessing normal distribution (2) using skewness and kurtosis. Restorative Dentistry \& Endodontics, 38(1), 52. https://doi.org/10.5395/rde.2013.38.1.52

Kujawa, A., Arfer, K. B., Klein, D. N., \& Proudfit, G. H. (2014). Electrocortical reactivity to social feedback in youth: A pilot study of the Island Getaway task. Developmental Cognitive Neuroscience, 10, 140-147. https://doi.org/10.1016/j.den.2014.08.008

Lapidot-Lefler, N., \& Barak, A. (2012). Effects of anonymity, invisibility, and lack of eyecontact on toxic online disinhibition. Computers in Human Behavior, 28(2), 434-443. https://doi.org/10.1016/j.chb.2011.10.014

Leary, M. R., Springer, C., Negel, L., Ansell, E., \& Evans, K. (1998). The causes, phenomenology, and consequences of hurt feelings. Journal of Personality and Social Psychology, 74(5), 1225-1237. https://doi.org/10.1037//0022-3514.74.5.1225 
Lobera, I. J., Estébanez, S., Fernández, M. J. S., Bautista, E. Á., \& Garrido, O. (2009). Coping strategies in eating disorders. European Eating Disorders Review, 17(3), 220-226. https://doi.org/10.1002/erv.920

MacLaren, V. V., \& Best, L. A. (2009). Female students' disordered eating and the big five personality facets. Eating Behaviors, 10(3), 192-195. https://doi.org/10.1016/j.eatbeh.2009.04.001

Milligan, R. J., \& Waller, G. (2000). Anger and bulimic psychopathology among nonclinical women. International Journal of Eating Disorders, 28(4), 446-450. https://doi.org/10.1002/1098-108X(200012)28:4<446::AID-EAT13>3.0.CO;2-J

Monteleone, A. M., Treasure, J., Kan, C., \& Cardi, V. (2018). Reactivity to interpersonal stress in patients with eating disorders: A systematic review and meta-analysis of studies using an experimental paradigm. Neuroscience and Biobehavioral Reviews, 87(December 2017), 133-150. https://doi.org/10.1016/j.neubiorev.2018.02.002

Mulkerrin, Ú., Bamford, B., \& Serpell, L. (2016). How well does Anorexia Nervosa fit with personal values? An exploratory study. Journal of Eating Disorders, 4(1), 1-11. https://doi.org/10.1186/s40337-016-0109-z

Nielsen, S., \& Bará-Carril, N. (2003). Narcissism and cardiovascular reactivity to rejection imagery. In J. Treasure, U. Schmidt, \& E. van Furth (Eds.), The Handbook of Eating Disorders (2nd ed., pp. 191-206). Wiley.

Peñas-Lledó, E., Fernández, J. D., \& Waller, G. (2004). Association of anger with bulimic and other impulsive behaviours among non-clinical women and men. European Eating Disorders Review, 12(6), 392-397. https://doi.org/10.1002/erv.588

Platt, J. J., \& Spivack, G. (1975). Manual for the Means-Ends Problem-Solving Procedure 
(MEPS): A measure of interpersonal cognitive problem-solving skill. Hahnemann Community Mental Heatlh/Mental Retardation Center.

Raykos, B. C., McEvoy, P. M., Carter, O., Fursland, A., \& Nathan, P. (2014). Interpersonal problems across restrictive and binge-purge samples: Data from a community-based eating disorders clinic. Eating Behaviors, 15(3), 449-452.

https://doi.org/10.1016/j.eatbeh.2014.06.008

Rayner, K. E., Schniering, C. A., Rapee, R. M., Taylor, A., \& Hutchinson, D. M. (2013). Adolescent girls' friendship networks, body dissatisfaction, and disordered eating: Examining selection and socialization processes. Journal of Abnormal Psychology, 122(1), 93-104. https://doi.org/10.1037/a0029304

Ridout, N., Matharu, M., Sanders, E., \& Wallis, D. J. (2015). The influence of eating psychopathology on autobiographical memory specificity and social problem-solving. Psychiatry Research, 228(3), 295-303. https://doi.org/10.1016/j.psychres.2015.06.030

Rieger, E., Van Buren, D. J., Bishop, M., Tanofsky-Kraff, M., Welch, R., \& Wilfley, D. E. (2010). An eating disorder-specific model of interpersonal psychotherapy (IPT-ED): Causal pathways and treatment implications. Clinical Psychology Review, 30(4), 400-410. https://doi.org/10.1016/j.cpr.2010.02.001

Roberts, M. E., Tchanturia, K., Stahl, D., Southgate, L., \& Treasure, J. (2007). A systematic review and meta-analysis of set-shifting ability in eating disorders. Psychological Medicine, 37(8), 1075-1084. https://doi.org/10.1017/S0033291707009877

Romero-Canyas, R., \& Downey, G. (2005). Rejection sensitivity as a predictor of affective and behavioral responses to interpersonal stress: A defensive motivational system. In K. D. Williams, J. P. Forgas, \& W. von Hippel (Eds.) The social outcast: Ostracism, social 
exclusion, rejection, and bullying (pp. 131-154). Psychology Press.

Russell, T. A., Schmidt, U., Doherty, L., Young, V., \& Tchanturia, K. (2009). Aspects of social cognition in anorexia nervosa: Affective and cognitive theory of mind. Psychiatry Research, 168(3), 181-185. https://doi.org/10.1016/j.psychres.2008.10.028

Schmidt, U., \& Treasure, J. (2006). Anorexia nervosa: Valued and visible. A cognitiveinterpersonal maintenance model and its implications for research and practice. British Journal of Clinical Psychology, 45(3), 343-366. https://doi.org/10.1348/014466505X53902

Snapp, C. M., \& Leary, M. R. (2001). Hurt feelings among new acquaintances: Moderating effects of interpersonal familiarity. Journal of Social and Personal Relationships, 18, 315326. https://doi.org/doi:10.1177/0265407501183001

Sommer, K. L., Kirkland, K. L., Newman, S. R., Estrella, P., \& Andreassi, J. (2009). Narcissism and cardiovascular reactivity to rejection imagery. Journal of Applied Social Psychology, 39, 1083-1115. https://doi.org/doi:10.1111/j.1559-1816.2009.00473.x

Spielberger, C. D. (1996). State-Trait Anger Expression Inventory: Professional manual. Psychological Assessment Resources.

Steinglass, J., \& Walsh, T. B. (2006). Habit learning and anorexia nervosa: A cognitive neuroscience hypothesis. International Journal of Eating Disorders, 39(4), 267-275. https://doi.org/10.1002/eat

Sternheim, L., Danner, U., van Elburg, A., \& Harrison, A. (2020). Do anxiety, depression, and intolerance of uncertainty contribute to social problem solving in adult women with anorexia nervosa? Brain and Behavior, 10(6), 1-11. https://doi.org/10.1002/brb3.1588

Stoeber, J., Madigan, D. J., Damian, L. E., Esposito, R. M., \& Lombardo, C. (2017). Perfectionism and eating disorder symptoms in female university students: the central role 
of perfectionistic self-presentation. Eating and Weight Disorders, 22(4), 641-648. https://doi.org/10.1007/s40519-016-0297-1

Suler, J. (2004). The online disinhibition effect. Cyberpsychology and Behavior, 7(3), 321-326. https://doi.org/10.1089/1094931041291295

Svaldi, J., Dorn, C., \& Trentowska, M. (2011). Effectiveness for interpersonal problem-solving is reduced in women with binge eating disorder. European Eating Disorders Review, 19(4), 331-341. https://doi.org/10.1002/erv.1050

Tchanturia, K., Morris, R. G., Anderluh, M. B., Collier, D. A., Nikolaou, V., \& Treasure, J. (2004). Set shifting in anorexia nervosa: An examination before and after weight gain, in full recovery and relationship to childhood and adult OCPD traits. Journal of Psychiatric Research, 38(5), 545-552. https://doi.org/10.1016/j.jpsychires.2004.03.001

Tchanturia, Kate, Davies, H., Harrison, A., Fox, J. R. E., Treasure, J., \& Schmidt, U. (2012). Altered social hedonic processing in eating disorders. International Journal of Eating Disorders, 45(8), 962-969. https://doi.org/10.1002/eat.22032

Tiller, J., Schmidt, U., Ali, S., \& Treasure, J. (1995). Patterns of punitiveness in women with eating disorders. International Journal of Eating Disorders, 17(4), 365-371. https://doi.org/10.1002/1098-108X(199505)17:4<365::AID-EAT2260170408>3.0.CO;2-A

Wildes, J. E., \& Marcus, M. D. (2011). Development of emotion acceptance behavior therapy for anorexia nervosa: A case series. International Journal of Eating Disorders, 44(5), 421-427. https://doi.org/10.1002/eat.20826

Wilfley, D. E., Stein, R., \& Welch, R. (2003). Interpersonal Psychotherapy. In J. Treasure, U. Schmidt, \& E. van Furth (Eds.), Handbook of Eating Disorders (2nd ed., pp. 253-270). Wiley. https://doi.org/10.1016/B978-0-08-097086-8.21065-9 
Zadro, L., Williams, K. D., \& Richardson, R. (2004). How low can you go? Ostracism by a computer is sufficient to lower self-reported levels of belonging, control, self-esteem, and meaningful existence. Journal of Experimental Social Psychology, 40(4), 560-567. https://doi.org/10.1016/j.jesp.2003.11.006 


\section{Table 1}

Descriptive Statistics and Zero-order Correlations

\begin{tabular}{lcccc}
\hline & 1 & 2 & 3 & 4 \\
\hline 1. Proportion of Rejection Votes & - & & & \\
2. Coplayer Liking & $-.34^{* *}$ & - & & \\
3. Binge Eating & .07 & .10 & & \\
4. Restricting & $.25^{* *}$ & .03 & .07 & - \\
\hline Mean & 0.39 & 0.11 & 9.12 & 6.41 \\
SD & 12.95 & 0.90 & 5.45 & 5.55 \\
Range & $0.06-0.67$ & $1.98-9.00$ & $0.00-29.00$ & $0.00-22.00$ \\
\hline
\end{tabular}

Note. Proportion of rejection votes includes voting behavior across all six rounds. 


\section{Table 2}

The Moderating Effect of ED Symptoms on the Association between Island Getaway Feedback and Participant Voting Behavior

\begin{tabular}{|c|c|c|c|c|c|}
\hline Predictor & Estimate & $S E$ & $p$ & Partial $R^{2}$ & $95 \%$ CIs \\
\hline \multicolumn{6}{|c|}{ Model 1: Binge eating as moderator } \\
\hline Intercept & -0.74 & 0.09 & $<.001$ & 0.62 & $-0.93,-0.54$ \\
\hline Coplayer liking previous round & -0.72 & 0.04 & $<.001$ & 0.07 & $-0.81,-0.63$ \\
\hline Previous round feedback & 0.56 & 0.12 & .001 & 0.66 & $0.29,0.82$ \\
\hline Binge eating & 0.12 & 0.07 & .11 & 0.02 & $-0.03,0.27$ \\
\hline Feedback $\mathrm{x}$ binge eating & -0.08 & 0.09 & .40 & 0.01 & $-0.27,0.11$ \\
\hline \multicolumn{6}{|c|}{$\underline{\text { Model 2: Dietary restriction as moderator }}$} \\
\hline Intercept & -0.74 & 0.09 & $<.001$ & 0.64 & $-0.94,-0.55$ \\
\hline Coplayer previous liking & -0.70 & 0.04 & $<.001$ & 0.08 & $-0.79,-0.62$ \\
\hline Previous round feedback & 0.55 & 0.12 & .001 & 0.65 & $0.29,0.82$ \\
\hline Dietary restriction & 0.16 & 0.07 & .03 & 0.09 & $0.01,0.30$ \\
\hline Feedback $\mathrm{x}$ dietary restriction & 0.05 & 0.10 & .58 & 0.002 & $-0.14,0.24$ \\
\hline
\end{tabular}

Note. Previous round feedback and participant voting are coded as $0=$ accept and $1=$ reject. 